\title{
Comparación de criterios de selección para ovinos Suffolk Down manejados en condiciones extensivas en la zona central de Chile
}

\author{
Selection criteria comparison for Suffolk Down sheep under range management in central Chile
}

\author{
F Lembeye $^{\mathrm{a}^{*}}$, G Castellaro ${ }^{\mathrm{b}}$, JC Magofke ${ }^{\mathrm{b}}$, H Uribe ${ }^{\mathrm{b}}$
}

${ }^{a}$ Institute of Veterinary, Animal and Biomedical Sciences, Massey University, Palmerston North, New Zealand.

${ }^{\text {b}}$ Departamento de Producción Animal, Facultad de Ciencias Agronómicas, Universidad de Chile, Santiago, Chile.

\section{SUMMARY}

\begin{abstract}
The aim of this study was to calculate selection indexes for a specialised meat sheep breed, Suffolk Down under range management in Central Chile. A selection index was constructed and relative economic weights for each trait were estimated. Genetic parameters were obtained from the literature and phenotypic parameters were estimated from the available data ( $N=330$ for PN and TC) of the Suffolk breed from the Estacion Experimental Rinconada de Maipu from the Universidad de Chile. Genetic-economic efficiency $(\Delta \mathrm{H})$ was estimated as a result of the use of indexes with and without constraints, and when selection is carried out to change a single trait. The traits included in the selection index $\left(\mathrm{I}_{\mathrm{S}}\right)$ were: birth weight $(\mathrm{PN})$ and birth to weaning growth rate (TC). The predicted genetic gain in PN and TC is minimal, due to the moderate to low heritability used ( 0.15 and 0.17 for PN and TC, respectively). In the simulations, TC was the trait that contributed most to the breeding goal. When PN is restricted, the expected loss is $20 \%$ regarding unrestricted selection index, meanwhile selection only for TC could be an effective alternative for sheep growers, since the loss is very small (6.28\%) regarding to the selection index and the correlated response on PN would be only $0.75 \%$ per generation, what could hardly result in dystocia increase.
\end{abstract}

Key words: selection indexes, sheep meat, relative efficiency.

\section{RESUMEN}

El objetivo del presente trabajo fue calcular índices de selección en ovinos especializados en producción de carne. Se estimaron las ponderaciones económicas relativas de cada característica considerada en el índice de selección, se obtuvieron parámetros genéticos bibliográficos y parámetros fenotípicos a partir de la información disponible ( $\mathrm{N}=330$ para eso al nacimiento (PN) y tasa de crecimiento (TC)) de los ovinos Suffolk de la Estación Experimental de Rinconada de Maipú de la Universidad de Chile. Se calculó la eficiencia genético-económica $(\Delta \mathrm{H})$, de la utilización de un índice con y sin restricciones y cuando la selección se hace sólo para una característica. El mejoramiento genético en PN y TC esperado es mínimo debido a las moderadas a bajas heredabilidades utilizados $(0,15$ y 0,17 para PN y TC, respectivamente). De las simulaciones realizadas, TC fue la característica que contribuyó en mayor medida al objetivo de mejoramiento. La selección restringiendo PN provocaría una pérdida de un $20 \%$ en relación al índice sin restricciones, mientras que la selección únicamente por TC podría ser una eficaz alternativa para los ganaderos, ya que la pérdida es muy pequeña en relación al índice de selección $(6,28 \%)$ y la respuesta correlacionada esperada en PN es únicamente $0,75 \%$ por generación, lo que difícilmente puede repercutir en un aumento en los partos distócicos.

Palabras clave: índices de selección, ovinos de carne, eficiencia relativa.

\section{INTRODUCCIÓN}

En Chile, el progreso genético logrado en producción ovina ha sido mayoritariamente consecuencia de la incorporación de reproductores de otros planteles sean estos importados o de una misma región; esto corresponde a migración y no a selección genética dentro del plantel. Por otra parte, en el país, han sido escasas las evaluaciones que informen objetivamente el progreso genético en planteles ovinos para producción de carne y/o lana. Una excepción a lo anterior es el trabajo realizado por Uribe y col (2011), del Instituto de Investigaciones Agropecuarias (INIA) en el Centro Experimental Hidango. Dichos

Aceptado: 21.11.2013.

* Private Bag 11,222, New Zealand; felipelembeye@gmail.com autores cuantificaron el avance genético logrado en ovinos para peso al nacer (PN) y al destete (PD) en el periodo 2003-2011, utilizando un modelo animal resuelto con metodología BLUP (Henderson 1950). El progreso logrado para PN en las razas Poll Dorset y Texel fueron: 230 y $45 \mathrm{~g}$, respectivamente, mientras que para PD este fue de 1.043 y $385 \mathrm{~g}$ en el mismo orden.

La característica económica de mayor importancia en ovinos de carne es kilos de cordero destetado por oveja encastada (García y col 2006), por tanto, ésta debiera ser el principal objetivo de selección (Safari y col 2006). Una desventaja de la selección por kilos de cordero destetado por oveja encastada es su baja heredabilidad, la cual ha sido documentada por Safari y col (2005) en 0,07 $\pm 0,01$.

Por otra parte, Hazel (1943) desarrolló una metodología para seleccionar animales superiores genéticamente 
denominada índice de selección. Este método permite hacer selección simultánea por dos o más características de distinta importancia económica. Esto es económicamente más eficiente comparado con selección genética basado en una sola característica. Aun así, la selección genética por una sola característica es el método más simple y generalmente entrega la mayor respuesta a la selección cuando las características presentan moderadas a altas heredabilidades (Cardellino y Rovira 1987, Falconer y Mackay 1996). Para caracteres con bajas heredabilidades, como es el caso de kilos de cordero destetado por oveja, la eficacia de la selección puede mejorarse sustancialmente al combinar la información fenotípica individual y de los parientes de los candidatos a reproductores (Conington y col 2001). Sin embargo, en la producción ovina tradicional en Chile se realiza monta natural usando varios carneros en un determinado lote de hembras por lo que se desconoce el origen paterno de los corderos, lo que impide hacer uso de métodos más eficientes de selección.

En Chile, las razones probables por las que el método de índice de selección no ha sido aplicado masivamente serían: 1- se requiere definir los objetivos de cría a nivel regional o nacional, 2- la dificultad de obtener de manera confiable los registros productivos en condiciones extensivas, 3- desconocimiento de los beneficios potenciales de esta metodología y 4- la dificultad de derivar adecuadamente valores económicos asociados a las características por la cuales se selecciona los animales (Yáñez 2005).

El objetivo de este trabajo fue comparar la efectividad de diferentes criterios de selección en ovinos de la raza Suffolk Down manejados en condiciones extensivas en la zona central de Chile.

\section{MATERIAL Y MÉTODOS}

Se construyeron diferentes índices y criterios de selección basados en información fenotípica de ovinos Suffolk Down pertenecientes a la Estación Experimental Rinconada de Maipú de la Facultad de Ciencias Agronómicas de la Universidad de Chile, ubicada en la comuna de Maipú, Región Metropolitana, Chile (3331' Lat. Sur; 7050’ Long. Oeste; 470 m.s.n.m.).

Las características a considerar en los índices de selección fueron peso al nacimiento (PN) y tasa de crecimiento (TC). Para dichas características se analizaron 330 registros con un promedio y coeficiente de variación (C.V.) de 4,5 kg y 20,7\% para PN y $284,8 \mathrm{~g} \mathrm{día}^{-1}$ y $17,6 \%$ para TC.

\section{ÍNDICE DE SELECCIÓN SIN RESTRICCIONES}

Para la construcción de un índice de selección, el primer paso es definir el objetivo de selección $(\mathrm{H})$, esto es, definir las características de importancia económica que interesan ser mejoradas del punto de vista genético (Simm 1998). En este trabajo, las características objetivo de selección fueron PN y TC. Por tanto, $\mathrm{H}$ es definido según la siguiente expresión:

$\mathrm{H}=a_{P N} G_{P N}+a_{T C} G_{T C}$

Donde $H$ es el merito genético o valor de cría agregado, $a_{P N}$ y $a_{T C}$ corresponden a las ponderaciones económicas de PN y TC, respectivamente. $G_{P N}$ y $G_{T C}$ corresponden a los valores genéticos de PN y TC. El objetivo de selección puede ser expresado en notación matricial como $a^{\prime}$ g y se denota por $H$. Un estimador de $H$ fue derivado por Hazel (1943), el cual es: $I=b^{\prime} p$, donde $I=$ índice de selección; $b^{\prime}=$ vector de ponderaciones a estimar $\mathrm{y}$ $p=$ vector de observaciones fenotípicas corregidas por factores no genéticos. Hazel (1943) demostró que este estimador maximiza la correlación entre $H$ e $I$, y minimiza la varianza de las diferencias entre $H$ e $I$, para mayor detalle de la igualdad $H=I$, consultar Cameron (1997). El índice de selección es el mejor estimador del verdadero valor de cría agregado de un animal $(\mathrm{H})$ y toma la forma de una regresión lineal múltiple (Falconer 1996). En este índice el valor fenotípico corregido de cada característica $\left(\mathrm{X}_{\mathrm{i}}\right)$ se pondera por un coeficiente $\left(\mathrm{b}_{\mathrm{i}}\right)$ que permite maximizar el beneficio genético-económico. Las expresiones para este trabajo se derivan de Cameron (1997):

$I=b_{P N} X_{P N}+b_{T C} X_{T C}$

Donde $I$ es el índice de selección. $b_{P N}$ y $b_{T C}$ corresponden a las ponderaciones de PN y TC, respectivamente. $X_{P N}$ y $X_{T C}$ corresponden a los valores fenotípicos de PN y TC, respectivamente, corregidos por otros efectos no genéticos.

Para la estimación de los valores de las ponderaciones del índice (b), se utilizó la siguiente igualdad:

$b=P^{-1} \cdot G \cdot a$

Donde $G$ es la matriz de (co)varianzas genéticas, $P^{-1}$ es la matriz inversa de varianzas y covarianzas fenotípicas, $a$ y $b$ son vectores definidos anteriormente para $H$ e $I$, respectivamente.

Los parámetros necesarios para la estimación de (co) varianzas genéticas requeridos en la construcción de índices de selección se obtuvieron de una recopilación realizada por Safari y Fogarty (2003) y su posterior resumen (Safari y col 2005). En relación a este punto, Safari y col (2006) señalan que un índice de selección calculado con parámetros genéticos y fenotípicos propios de la población, nunca es menos eficientes que cuando se utilizan parámetros de la literatura. Sin embargo, cuando los parámetros no están disponibles para la misma población, los autores mencionados anteriormente, indican que en la práctica no hay inconvenientes importantes para calcular un índice basado en parámetros de otra población estimados para una misma raza o línea y manejada en condiciones ambientales similares. 
Las covarianzas fenotípicas $\left(\sigma_{P_{X Y}}\right)$ se obtuvieron multiplicando la correlación fenotípica $\left(r_{P_{X Y}}\right)$ por las desviaciones estándar de los caracteres $\mathrm{X}\left(\sigma_{x}\right)$ e Y $\left(\sigma_{y}\right)$ :

$\sigma_{\mathrm{P}_{\mathrm{XY}}}=\mathrm{r}_{\mathrm{P}_{\mathrm{XY}}} \cdot \sigma_{\mathrm{X}} \cdot \sigma_{\mathrm{Y}}$

Se utilizó una desviación estándar fenotípica $\sigma_{P}$ de: $0,76 \mathrm{~kg}$ y $33,9 \mathrm{~g} \mathrm{día}^{-1}$, para PN y TC, respectivamente (Cuadro 1) y una correlación fenotípica $r_{P_{X Y}}$ de $0,12^{1}$.

La varianza $\left(\sigma_{G}{ }^{2}\right)$ y desviación estándar $\left(\sigma_{G}\right)$ genética necesarias para el cálculo del índice de selección, se obtuvieron a través de la siguiente expresión (Cameron 1997):

Varianza genética aditiva:

$\sigma_{\mathrm{G}}^{2}=\mathrm{h}^{2} \cdot \sigma_{\mathrm{P}}^{2}$

Donde: $h^{2}=$ heredabilidad y $\sigma_{P}^{2}=$ varianza fenotípica.

Las covarianzas genéticas $\left(\sigma_{G_{X Y}}\right)$ se obtuvieron a través de la siguiente expresión:

$\sigma_{G_{X Y}}=r_{G_{X Y}} \cdot \sqrt{h_{X}^{2} \cdot \sigma_{P_{X}}^{2}} \sqrt{h_{Y}^{2} \cdot \sigma_{P_{Y}}^{2}}$

Donde $r_{G_{X Y}}$ es la correlación genética entre las variables X e Y. $\sqrt{h^{2} \cdot \sigma_{P_{1}}^{2}}$ es la desviación estándar genética de la variable $\mathrm{X}$ o $\mathrm{Y}$.

Se calculó además la varianza del índice de selección, por cada unidad de intensidad de selección (is $=1$ ), a través de la siguiente expresión (Cameron 1997):

$\sigma_{1}^{2}=b^{\prime} \cdot \mathrm{P} \cdot \mathrm{b}$

Donde $b^{\prime}=$ es el vector $\mathbf{b}$ transpuesto.

El cambio estimado en cada una de las características consideradas en el índice $\left(\Delta_{i}\right)$ se calculó según la siguiente fórmula (Hogsett y Nordskog 1958):
$\Delta_{i}=\frac{b_{1} \cdot g_{i 1}+b_{2} \cdot g_{i 2}+\ldots b_{n} \cdot g_{i n}}{\sigma_{1}}$

Los valores $\mathrm{g}_{\mathrm{i} 1}, \mathrm{~g}_{\mathrm{i} 2}, \ldots \mathrm{g}_{\mathrm{in}}$ son los elementos correspondientes al carácter $\boldsymbol{i}$ de la matriz de varianzas y covarianzas genéticas $\mathrm{G}$.

Se determinó la eficiencia económica del uso del índice de selección como un cambio en valor de cría agregado $(\Delta H)$, después de una generación, con una intensidad de selección igual a 1 con la siguiente ecuación (Conington y col 2001)

$\Delta H=a_{F N} \Delta_{F N}+a_{T C} a_{T C}$

Donde $\Delta H$ es la ganancia económica después de una generación de selección al utilizar el índice de selección y que considera la sumatoria del cambio genético logrado en PN y TC multiplicadas por la ponderación económica dada a cada una de las variables lo que corresponde a la desviación estándar del índice de selección (Conington y col 2001).

\section{CÁLCULO DE LAS PONDERACIONES ECONÓMICAS}

En la ecuación 1 se define un vector $a$ como aquel que contiene las ponderaciones económicas de las características incluidas en el índice de selección. La ponderación económica de una característica es definida como el incremento en el ingreso neto $(\Delta \mathrm{IN})$ por unidad de cambio del carácter $\left(\Delta_{i}\right)$, manteniendo las otras características constantes (García y Gallardo 2005).

Para la valorización económica de PN y TC se consideraron los siguientes supuestos: (i) precio en pie del kilogramo de cordero corresponde al precio promedio pagado a productor en feria. El promedio utilizado fue \$737 pesos chilenos y se obtuvo de los registros históricos (1990-2011) correspondientes al mes de octubre, por ser el mes en el cual se concentra la venta de corderos en la zona central ${ }^{2}$. (ii) los animales son manejados en con-

Cuadro 1. Heredabilidad $\left(h^{2}\right)$, varianza $\left(\sigma^{2}\right)$ y desviación estándar $(\sigma)$ fenotípica y genética, ponderaciones económicas relativas $(a)$, correlaciones $(r)$ y covarianzas (Cov) fenotípica y genética entre peso al nacer (PN) y tasa de crecimiento (TC) en ovinos Suffolk.

Heritability $\left(h^{2}\right)$, phenotypic and genetic variance $\left(\sigma^{2}\right)$ and standard deviation $(\sigma)$, relative economic weights $(a)$, correlations $(r)$ and phenotypic and genetic co variances (Cov) between birth weight (PN) and growth rate (TC) in Suffolk sheep.

\begin{tabular}{|c|c|c|c|c|c|c|c|c|c|}
\hline & $\sigma_{\mathrm{P}}^{2}$ & $\sigma_{\mathrm{P}}$ & $\mathrm{h}^{2}$ & $\sigma_{\mathrm{A}}$ & $a^{(1)}$ & $\underset{\mathrm{PN}-\mathrm{TC}}{\mathrm{r}_{\mathrm{p}}}$ & $\begin{array}{l}\operatorname{Cov}_{P} \\
\text { PN-TC }\end{array}$ & $\begin{array}{c}\mathrm{r}_{\mathrm{G}} \\
\text { PN-TC }\end{array}$ & $\begin{array}{l}\mathrm{Cov}_{G} \\
\text { PN-TC }\end{array}$ \\
\hline \multirow[t]{2}{*}{ PN } & 0,58 & 0,76 & 0,15 & 0,30 & 13,5 & & & & \\
\hline & & & & & & 0,12 & 3,09 & 0,27 & 1,13 \\
\hline $\mathrm{TC}$ & 1.150 & 33,91 & 0,17 & 13,98 & 1,0 & & & & \\
\hline
\end{tabular}

${ }^{(1)}$ Se dividió el incremento neto marginal calculado por la característica TC para obtener ponderaciones relativas.

$h^{2}$ y $r_{G}$ corresponden a las informadas por Safari y col (2005).

1 Lembeye y col 2013, datos no publicados.

2 ODEPA 2011, http://www.odepa.gob.cl/ServletSeriesPreciosScr;j sessionid=DD91E7D996DC1C08C02514C5E7BC1CE1 ?modulo $=4 \&$ menu=precios $\&$ item $=$ rubros $\&$ rubro=pecuario 
diciones extensivas siendo alimentados únicamente con el pastizal natural mediterráneo.

Ponderación económica para tasa de crecimiento. Para calcular el ingreso marginal del aumento en la TC, se calculó el costo económico en que debe incurrir el productor para aumentar en una unidad la TC. Para ello se valorizó el kilogramo de materia seca (MS) con la siguiente ecuación (Daza 1997):

$C_{g}=\frac{E+M \cdot(n-1)}{n \cdot P}$

Donde $C_{g}$ es el costo anual del kilógramo de $\mathrm{MS}\left(\$ \mathrm{~kg}^{-1}\right)$; $E$ es el costo de establecimiento de la pradera $\left(\$ \mathrm{ha}^{-1}\right) ; M$ es el costo anual de mantención de la misma $\left(\$ h^{-1} \mathrm{año}^{-1}\right)$; $n$ es el número de años que dura la pradera en el predio y $P$ es la producción promedio de MS de la pradera $\left(\mathrm{kg} \mathrm{ha}^{-1}\right)$.

Por tratarse de un pastizal natural, no se consideraron costos de establecimiento, y debido a que usualmente no se realiza fertilización ni otros manejos en la pradera, el costo de mantención de la misma se supuso equivalente al $10 \%$ de los costos asociados a la mantención de cercos y arreglo de los espinos (Acacia caven) del predio en un año. La producción anual promedio de MS de la pradera se supuso ${ }^{3}$ en $1.100 \mathrm{~kg} \mathrm{ha}^{-1}$ y su duración en el predio se supuso en 20 años.

Se supuso que el consumo adicional de MS que implica aumentar en un kilo el peso al destete es $6,1 \mathrm{~kg}$ de MS ya que aumentar un kilo de peso vivo al destete demanda, según Nicol y Brookes (2007), un requerimiento de 58 MJ de EM y la concentración energética del pastizal natural es de 9,5 $\mathrm{MJ} \mathrm{kg}^{-1}$ de EM para la época de destete (Ovalle y Squella 1996). Según la ecuación 10, se valorizó el costo económico que representa dicho consumo. Dado el valor económico de cada gramo de cordero (supuesto 1), se calculó el ingreso neto de cada gramo de TC como la diferencia entre el valor de cada gramo y el costo económico que implica que el cordero crezca a una tasa diaria de un gramo mayor. Posteriormente, la ponderación económica de la variable TC se obtuvo como el producto del valor neto de cada gramo y la edad promedio al destete para determinar el ingreso neto durante todo el periodo de crecimiento.

Ponderación económica para peso al nacimiento. Su importancia económica se obtuvo a través de la correlación fenotípica $\left(\mathrm{r}_{\mathrm{P}_{\mathrm{PN}-\mathrm{PD}}}\right)$ positiva que entre $\mathrm{PN}$ y peso al destete (PD). La correlación fenotípica se transformó en regresión fenotípica $\left(\mathrm{b}_{\mathrm{PD}-\mathrm{PN}}\right)$ a través de la siguiente expresión (Cameron 1997):

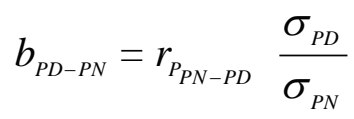

Alfredo Olivares, 2012. Profesor Titular Facultad Ciencias Agropecuarias, Universidad de Chile. Comunicación personal.
Donde $\sigma_{\mathrm{PN}}$ y $\sigma_{\mathrm{PD}}$ corresponden a las desviaciones estándar fenotípicas de PN y PD, respectivamente. Regresando el peso al destete sobre el peso al nacimiento se pudo estimar las unidades de cambio en el peso al destete por cada unidad de aumento en el peso al nacer.

Selección a favor de una característica. Se calculó también la respuesta directa a la selección $\left(R_{i}\right)$ en una generación sobre la base de seleccionar por una característica considerando una intensidad de selección (is) igual a una desviación estándar. Para este cálculo se utilizó la siguiente fórmula (Cameron 1997):

$\mathrm{R}=$ is $\cdot \sigma_{\mathrm{P}_{\mathrm{i}}} \cdot \mathrm{h}^{2}$

Donde $R_{i}$ es la respuesta a la selección para la característica $i$. is es la intensidad de selección. $\sigma_{\mathrm{P}_{\mathrm{i}}}$ es la desviación estándar fenotípica de la característica $i, \mathrm{~h}_{\mathrm{i}}^{2} \mathrm{y}$ corresponde a la heredabilidad de la característica $i$.

Los cambios que se esperan en la otra variable como consecuencia de la respuesta directa, se obtuvieron a través de la respuesta correlacionada (Cameron 1997):

$R C_{j}=i S_{i} \cdot r_{G_{i j}} \cdot h \cdot h_{j} \cdot \sigma_{P_{j}}$

Donde $R C_{j}$ es la respuesta indirecta de la característica $j$ al realizar selección directa por la característica $i$. $i s_{i}$ es la intensidad de selección utilizada al seleccionar por $i$. $\mathrm{r}_{\mathrm{Gij}}$ es la correlación genética entre $i$ y $j . h_{i}$ es la raíz cuadrada de la heredabilidad de la característica $i . h_{j}$ es la raíz cuadrada de la heredabilidad de la característica j, y $\sigma_{\mathrm{P}}$ corresponde a la desviación estándar fenotípica de la característica $j$.

Índice de selección con restricciones. En este trabajo se simuló la ganancia genética económica cuando se utiliza un índice de selección restringiendo a cero el cambio en PN ó TC. Para ello se utilizó la metodología descrita por Kempthorne y Nordskog (1959):

$b=\left[I^{*}-P^{-1} \cdot G^{*} \cdot\left(G^{*} \cdot P^{-1} \cdot G^{*}\right)-1 \cdot G^{*}\right] \cdot P^{-1} \cdot G \cdot a$

Dónde $b$ corresponde al vector de ponderaciones desconocidas a estimar. I* es la matriz de identidad. $G^{*}$ corresponde a las hileras de $G$ para las variables cuyo cambio será igual a cero y $G^{*}$ ' es la matriz $G^{*}$ traspuesta.

Eficiencia relativa. Dado que con un índice de selección sin restricciones se obtiene el mejoramiento genético que aporta el mayor beneficio genético económico, cualquier otro criterio de selección necesariamente es menos eficiente (Willis 1998). En función de lo anterior la eficiencia relativa (ER) de cualquier otro criterio de selección se obtuvo a través de la siguiente relación:

$\mathrm{ER}=\frac{\Delta \mathrm{H}\left(\mathrm{I}_{\mathrm{I}}\right)}{\Delta \mathrm{H}\left(\mathrm{I}_{\mathrm{S}}\right)} \cdot 100$

Donde $\Delta H\left(I_{I}\right)$ corresponde a la ganancia genéticoeconómica que se obtiene con un índice de selección con 
restricciones o a favor de una sola característica y $\Delta H\left(I_{S}\right)$ corresponde a la ganancia genético-económica que se obtiene con el índice de selección sin restricciones. La eficiencia relativa permitió comparar los diferentes índices de selección usados en este trabajo.

\section{RESULTADOS}

\section{ESTIMACIÓN DE LAS PONDERACIONES ECONÓMICAS RELATIVAS}

Tasa de crecimiento. De acuerdo a la ecuación 10, el costo de un kilo de materia seca en la Estación Experimental Rinconada de Maipú es de \$2,7 (datos no mostrados). El consumo adicional de $6,1 \mathrm{~kg}$ de materia seca del par oveja-cordero representa por tanto un costo de $\$ 16,5$. El valor neto de cada gramo en la tasa diaria de crecimiento es de $\$ 0,72$, si se considera que la edad promedio al destete fue 99,7 días, el aumento de un gramo en la tasa de crecimiento representa un aumento de \$71,9.

Peso al nacer. Se determinó el valor económico de PN como el peso adicional al destete a consecuencia del aumento de un kilo de peso al nacer. El valor del coeficiente de regresión (ecuación 11) fue de 1,351 lo que significa un ingreso de $\$ 995,7$. El incremento de 1,351 kilos adicionales al destete implica que el par oveja-cordero requiere consumo extra de $8,2 \mathrm{Kg} / \mathrm{MS}$. Este consumo extra representa un costo adicional de $\$ 22,1$; por consiguiente el ingreso neto marginal es $\$ 973,6$.

\section{CRITERIOS DE SELECCIÓN Y PÉRDIDA DE EFICIENCIA EN RELACIÓN AL ÍNDICE SIN RESTRICCIONES}

Índice de selección sin restricciones $\left(I_{S}\right)$. En la población de la cual se obtuvieron los parámetros fenotípicos, no existe actualmente incidencia de partos con problemas debido a altos pesos al nacimiento, por lo tanto, en una primera etapa, la selección para ovinos Suffolk puede orientarse a tratar de incrementar ambas características simultáneamente. En el cuadro 1 se señalan, para PN y TC, los parámetros genéticos, fenotípicos y económicos utilizados en el cálculo del índice de selección.

En el cuadro 2 se muestra el valor de los coeficientes de ponderación (b), la respuesta que se espera en cada carácter de la función objetivo por generación $\left(\Delta_{P N}, \Delta_{T C}\right)$ y la ER luego de seleccionar los animales que están a una desviación estándar sobre el promedio.

Con la utilización del índice de selección sin restricciones, se lograrían teóricamente cambios deseables en ambas características de interés y la mayor ganancia genético económica $\left(\Delta_{\mathrm{H}}\right)$. Al usar un índice sin restricciones con una intensidad de selección de una desviación estándar se espera un mejoramiento genético por generación de $70 \mathrm{~g}$ en PN y 5,6 $\mathrm{g} \mathrm{día}^{-1}$ en TC (cuadro 2). Este aumento se logra debido a la correlación genética positiva y favorable entre ambas variables.

Selección directa por PN y TC. Como se observa en el cuadro 2, la mayor ganancia genética para cada característica en forma independiente, se obtuvo cuando se simuló la selección exclusiva de cada una de ellas. La ventaja antes señalada es, sin embargo, muy pequeña para TC ya que la ganancia esperada por selección directa es $5,76 \mathrm{~g} \mathrm{día}^{-1}$, mientras que con el $\mathrm{I}_{\mathrm{S}}$ se espera una ganancia de 5,68 $\mathrm{g} \mathrm{día}^{-1}$. Para PN sin embargo, la ventaja de la selección directa es mucho mayor que la que se obtiene con el índice sin restricciones, ya que su valor es $71.4 \%$ superior (cuadro 2). Es importante destacar también que la eficiencia de la selección cuando se selecciona sólo por TC es 93,72\% comparada con el uso del índice de selección sin restricciones.

Índice con restricciones Los resultados que se informan en el cuadro 2 muestran que al restringir PN se pierde un $20 \%$ de eficiencia, comparado con un índice sin restricción, en cambio al hacerlo con TC la pérdida de eficiencia es de un 78,3\%.

Cuadro 2. Coeficientes de ponderación $\left(b_{i}\right)$, ganancia genética $\left(\Delta G_{i}\right)$, mejoramiento genético económico $(\Delta H)$ y eficiencia relativa obtenido por generación con una intensidad de selección igual a 1, al usar un índice de selección sin restricciones $\left(\mathrm{I}_{S}\right)$, con restricción y al hacer selección a favor de una sola característica.

Weights $\left(b_{i}\right)$, genetic gain, $\left(\Delta G_{i}\right)$, economic genetic improvement $(\Delta H)$ and relative efficiency after one generation with a selection intensity equal to 1 , by using selection indexes with and without restriction $\left(I_{S}\right)$, and selection for a single trait.

\begin{tabular}{|c|c|c|c|c|c|c|c|c|c|}
\hline \multirow{2}{*}{ Índice } & \multirow{2}{*}{$\begin{array}{l}\text { Tipo de } \\
\text { restricción }\end{array}$} & \multirow{2}{*}{$\mathrm{b}_{\mathrm{PN}}$} & \multirow{2}{*}{$\mathrm{b}_{\mathrm{TC}}$} & \multicolumn{2}{|c|}{$\triangle P N$} & \multicolumn{2}{|c|}{$\Delta T C$} & \multirow{2}{*}{$\Delta H$} & \multirow{2}{*}{$\begin{array}{c}\text { Eficiencia } \\
\text { relativa (\%) }\end{array}$} \\
\hline & & & & $(\mathrm{kg})$ & $(\%)$ & $\left(\mathrm{g} \mathrm{d}^{-1}\right)$ & $(\%)$ & & \\
\hline $\mathrm{I}_{\mathrm{S}}$ & Ninguna & 3,045 & 0,175 & 0,07 & 1,58 & 5,68 & 2,04 & 6,63 & 100 \\
\hline $\mathrm{I}_{\mathrm{PN}}$ & $\mathrm{PN}$ & $-1,979$ & 0,155 & 0,00 & 0,00 & 5,30 & 1,91 & 5,30 & 80,00 \\
\hline $\mathrm{I}_{\mathrm{TC}}$ & $\mathrm{TC}$ & 1,873 & $-0,011$ & 0,11 & 2,40 & 0,00 & 0,00 & 1,44 & 21,66 \\
\hline Sólo PN & & 1 & & 0,12 & 2,59 & 1,46 & 0,53 & 3,01 & 45,49 \\
\hline SóloTC & & & 1 & 0,03 & 0,75 & 5,76 & 2,07 & 6,21 & 93,72 \\
\hline
\end{tabular}

$\mathrm{I}_{S}$ : Índice de selección sin restricciones; $\mathrm{I}_{P N} \mathrm{I}_{T C}$ : Índice de selección cuando se restringe peso al nacer y tasa de crecimiento, respectivamente. 


\section{DISCUSIÓN}

El principal interés práctico en la utilización de un índice con restricciones es mantener el peso al nacimiento sin cambio genético. La otra opción, esto es restringir $\mathrm{TC}$, sólo tiene un interés académico, ya que obviamente las explotaciones comerciales de ovinos de carne tienen interés en aumentar sus tasas de crecimiento para destetar corderos de mayor peso.

En el índice sin restricciones, ambos ponderadores fueron positivos, lo que significa según Cardellino y Rovira (1987), que el índice otorga mayor puntaje a aquellos animales que tanto, el PN como la TC, tengan mérito genético superior a la media de la población. Distinto es el caso cuando se utiliza un índice restringiendo el aumento de PN ó TC, los coeficientes de ponderación $b_{P N} \mathrm{y}$ $b_{T C}$ tienen, según el caso, signo negativo al restringir PN y TC, respectivamente. Esto se debe a la necesidad de favorecer a los individuos con mérito genético para pesos al nacer o ganancia de peso, iguales o inferiores a la media de la población cuando uno de estos caracteres se restringen. Estos resultados concuerdan con los obtenidos por Winder y col (1990), quienes también dedujeron coeficientes de ponderación negativos cuando se restringe $\mathrm{PN}$ en bovinos de carne.

La alta importancia de TC en la ganancia genéticoeconómica obtenida en este estudio, que se refleja en el cuadro 2 donde el índice de selección incluyendo solo TC alcanza un $93,72 \%$ de efectividad comparado con un índice sin restricción, es congruente con los resultados de un trabajo de simulación realizado en la raza Targhee por Snowder y Van Vleck (2003). Los autores señalan que los esquemas de selección que favorecen una mayor ganancia de peso en corderos (pre y post destete) tienen a su vez la mayor influencia sobre la respuesta genética y económica. En dicho trabajo, se construyeron dos índices de selección. Índice I, que incluyó las características ganancia de peso post destete, peso corporal (200 días aproximadamente) y consumo de alimento; e Índice II, que incluyó peso corporal.

Givens y col (1960), también simularon selección directa por TC comparada con un índice de selección que consideró TC y calidad de canal. Los investigadores obtuvieron similar eficiencia con estas dos opciones.

En términos porcentuales, por selección directa se obtendría una mayor ganancia en PN que en TC, a pesar de que para TC se consideró una mayor heredabilidad (cuadro 1). La respuesta a la selección depende también de la variabilidad $\underset{(\mathrm{P})}{\sigma}$ de la característica. En este estudio, PN presentó un mayor C.V. que TC lo que podría explicar estos resultados (Cardellino y Rovira 1987).

En relación a los índices con restricciones, la restricción de PN produce una mayor reducción en la eficiencia respecto a la selección directa por TC. Esto se explica por la correlación genética positiva que existe entre estas dos variables ya que el valor de las heredabilidades utilizadas fue similar (cuadro 1). No obstante, es importante señalar que al hacer selección sólo por TC, se obtiene una eficiencia 13,7 puntos porcentuales superior respecto a la restricción de PN. Además, la respuesta correlacionada de PN al realizar selección sólo por TC es muy baja (33 g que equivale a un incremento de $0,75 \%$ por generación) (cuadro 2). Dado los resultados de la simulación se demuestra que, en teoría, la selección sólo por TC no provocaría un incremento suficientemente importante en PN como para generar problemas de partos distócicos en el mediano plazo.

En la literatura revisada en este trabajo no se han publicado reportes experimentales de selección restringiendo PN en ovinos; sin embargo, Winder y col (1990), Kahi y Hirooka (2007) y Melucci y col (2009), demostraron que es posible aumentar la TC y PD sin aumentar el PN en bovinos de carne. Estos resultados son factibles porque existe una alta variación genética para el crecimiento que es independiente del PN (Winder y col 1990). Koch y col (1974) en bovinos de la raza Hereford, informan que se podría reducir cerca de un $30 \%$ del incremento de PN si la selección se realiza por ganancia de peso en vez de peso al destete o peso adulto.

De acuerdo a los resultados obtenidos en este trabajo, en cualquier otro criterio de selección a excepción de seleccionar sólo por TC, se reduce la eficiencia en magnitudes superiores al $20 \%$ en comparación a utilizar el índice sin restricciones (cuadro 2). Debido a las complicaciones propias para la construcción de un índice de selección; usar tasa de crecimiento como único criterio de selección podría ser una alternativa recomendada.

El mejoramiento anual de PN y TC por selección individual es pequeño debido a que en condiciones de pastoreo extensivo, el valor fenotípico de los individuos está influido por componentes ambientales que no son heredables (Winder y col 1990, Snyman y col 1995). De acuerdo a los resultados obtenidos por Lasslo y col (1985) en ovinos seleccionados a los $120 \mathrm{~d}$ por un período de 20 años, se obtuvo un aumento en peso en la progenie de los animales seleccionados en comparación al rebaño control. Esta diferencia, sin embargo, no fue estadísticamente significativa. La principal razón se debió a fluctuaciones anuales en las condiciones medioambientales durante el período evaluado y al bajo índice de herencia realizado dada estas condiciones $\left(\mathrm{h}^{2}: 0,064 \pm 0,02\right)$. Lo anterior es compartido por Rodríguez y col (2004), quienes informan que la selección masal aplicada en ovinos Sugureños ha sido ineficaz en aumentar la tendencia genética para características de peso y crecimiento.

Debido a la gran influencia materna con caracteristicas pre-destete, la selección de reproductores podría ser más efectiva si se utilizara como criterio de selección el peso post destete o peso adulto, debido a que estas características poseen una menor influencia materna, y por este motivo los índices de herencia son más altos (Snyman y col 1995, Notter y Hough 1997, Snowder y Van Vleck 2003). Sin embargo, la necesidad de seleccionar 
animales a edades más tempranas dificulta esta práctica (Lax y col 1979). Mavrogenis y Constantinou (1990) y Sakul y col (1994) concuerdan que la selección por peso post destete incrementaría el peso adulto, lo que traería como consecuencia un aumento de los requerimientos energéticos de mantención. Por tanto, recomiendan que la selección se realice a edades más tempranas. Esta afirmación también sería válida para bovinos de carne.

A consecuencia de lo señalado en el párrafo anterior, Rodríguez y col (2004) señalan que en ovinos de carne se debe tratar de conseguir mejoras en los pesos y crecimiento sin aumentar el peso adulto. A pesar de ello, teóricamente, la selección por PN y TC traería como consecuencia respuestas correlacionadas sobre peso post destete y peso adulto debido a correlaciones genéticas positivas. Safari y col (2005), señalan una $r_{G}$ entre PN con peso post-destete y peso adulto de: 0,29 y 0,22 , respectivamente y una $r_{G}$ entre TC con peso post-destete y peso adulto de 0,19 y 0,78 , respectivamente. En este punto, sin embargo, no existiría una ventaja en seleccionar por TC en vez de PD con el propósito de evitar un mayor peso adulto, ya que la correlación genética entre PD con peso adulto informada por Safari y col (2005) $\left(r_{G}: 0,75\right)$ es muy similar a la $r_{G}$ entre TC y peso adulto.

En relación a evaluaciones genéticas en ovinos para carne, Rodríguez y col (2004) sugieren que los ganaderos debieran utilizar valores de cría obtenidos por la metodología BLUP que considera el comportamiento individual y familiar, lo que unido a una correcta dirección técnica en la toma de decisiones redunda sin duda en un eficaz progreso genético.

Teóricamente es factible mejorar ambas características en ovinos Suffolk, sin embargo, este incremento es pequeño en condiciones extensivas ya que las heredabilidades, como se reportan en la literatura, son moderadas a bajas para PN y TC. Es importante señalar que la selección directa solo por TC posee una alta eficiencia en relación al índice sin restricciones, por tanto, en condiciones prácticas para ovinos de carne, se recomienda que la selección se oriente a esta característica; lo anterior se justifica dada las dificultades de construir un índice de selección. Además, la selección solamente por TC tendría un escaso impacto sobre PN, esto resguardando la posibilidad de partos distócicos. Para este último caso, la selección restringiendo PN también podría ser recomendada pero la eficiencia es menor que la selección directa para TC en relación al índice sin restricciones.

\section{REFERENCIAS}

Cameron N. 1997. Selection indices and prediction of genetic merit in animal breeding. CAB International, Wallingford, UK.

Cardellino R, J Rovira. 1987. Mejoramiento genético animal. Editorial Agropecuaria Hemisferio Sur, Montevideo, Uruguay.
Conington J, S Bishop, B Grundy, A Waterhouse, G Simm. 2001. Multi-trait selection indexes for sustainable UK hill sheep production. J Anim Sci 73, 413-423.

Daza A. 1997. Reproducción y Sistemas de Explotación del ganado ovino. Editorial Mundi Prensa, Madrid, España.

Falconer DS, MT Mackay. 1996. Introduction to quantitative genetics. $4^{\text {th }}$ ed. Essex, UK.

García X, J Gallardo. 2005. Predictores del valor de cría: índices de selección y metodología de los modelos mixtos. Publicación Docente $\mathrm{N}^{\circ} 17$. Departamento de Producción Animal, Fac. de Cs. Agrarias y Forestales, Universidad de Chile, Santiago, Chile.

García X, JC Magofke, C Aubert. 2006. Comportamiento productivo del Merino Precoz y Suffolk en el secano interior de la Región Metropolitana, Chile. Avances en Producción Animal 31, 35-56.

Givens C, R Carter, J Gaines. 1960. Selection indexes for weaning traits in spring lambs. J Anim Sci 19, 134-139.

Hazel L. 1943. The genetic basis for constructing selection indexes. Genetics 28, 476-490.

Henderson C. 1950. Estimation of genetic parameters. Ann Math Stat 21, 309-310.

Hogsett M, A Nordskog. 1958. Genetic-economic value in selecting for egg production rate, body weight and egg weight. Poultry Sci 37, 1404-1419.

Kahi A, H Hirooka. 2007. Effect of direct and indirect selection criteria for efficiency of gain on profitability of Japanese Black cattle selection strategies. J Anim Sci 85, 24012412.

Kempthorne O, A Nordskog. 1959. Restricted selection indexes. Biometrics 15, 10-19.

Koch R, K Gregory, L Cundiff. 1974. Selection in beef cattle II Selection Response. J Anim Sci 39, 459-470.

Lasslo L, G Bradford, D Torell, B Kennedy. 1985. Selection for weaning weight in Targhee sheep in two environments. I. Direct Response. J Anim Sci 61, 376-386.

Lax J, A Chapman, A Pope, R Baker, B Bradley. 1979. Comparison of single trait and index selection in sheep. $J$ Anim Sci 48, 776-788.

Mavrogenis A, A Constantinou. 1990. Relationship between pre-weaning growth, post-weaning growth and mature body size in Chios sheep. Anim Prod 50, 271-275.

Melucci L, A Birchmeir, E Cappa, J Cantet. 2009. Bayesian analysis of selection for greater weaning weight while maintaining birth weight in beef cattle. J Anim Sci 87, 3089-3096.

Nicol A, I Brookes. 2007. The metabolizable energy requirements of grazing livestock. In: Rottary P, Brookes I, Nicol A (eds). Pasture and supplements for grazing animals. New Zealand Society of Animal Production, Occasional Publication No. 14. Pp 151-172.

Notter D, J Hough. 1997. Genetic parameter estimates for growth and fleece characteristics in Targhee sheep. J Anim Sci 75, 1729-1737.

Ovalle C, F Squella. 1996. Terrenos de pastoreo con pastizales anuales en el área de influencia climática mediterránea. En: Ruiz I (ed). Praderas para Chile. $2^{\mathrm{a}}$ ed. Instituto de Investigaciones Agropecuarias, Santiago, Chile, Pp 429-466.

Rodríguez J, J Delgado, J Quiroz, J León. 2004. Evaluación de la tendencia genética en la raza ovina Sugureña bajo selección masal por características de crecimiento. IV Con- 
greso Ibérico sobre Recursos Genéticos Animáis, Ponte de Lima, Septiembre Portugal.

Safari E, N Fogarty. 2003. Genetic parameters for sheep production traits. NSW Agriculture Technical Bulletin No. 49. NSW Agriculture, Orange Agricultural Institute, Orange, Australia.

Safari E, N Fogarty, A Gilmour. 2005. A review of genetic parameter estimates for wool, growth, meat and reproduction traits in sheep. Livest Prod Sci 92, 271-289.

Safari E, N Fogarty, A Gilmour. 2006. Sensitivity of response of multi-trait index selection to changes in genetic correlations between production traits in sheep. Aus J Exp Agric 46, 283-290.

Sakul H, G Bradford, M Dally, T Famula, C Finley. 1994. Growth rate in sheep selected for weaning weight or litter size in a range environment. Proc. $5^{\text {th }}$ World Congress Genet Appl Livest Ontario, Canada, 18, 59-62.

Simm G. 1998. Genetic improvement of Cattle and Sheep. Farming Press, UK.

Snowder G, L Van Vleck. 2003. Estimates of genetic parameters and selection strategies to improve the economic effi- ciency of postweaning growth in lambs. J Anim Sci 81, 2704-2713.

Snyman M, G Erasmus, J Van Wyk, J Olivier. 1995. Direct and maternal (co)variance components and heritability estimates for body weight at different ages and fleece traits in Afrino Sheep. Livest Prod Sci 44, 229-235.

Uribe H, F Squella, R de la Barra, M Martínez. 2011. Cuantificación del cambio genético para pesos al nacimiento y destete en ovinos Dorset y Texel. XXXVI Reunión Anual Sociedad Chilena de Producción Animal, Punta Arenas, Chile, Pp 7-8.

Willis M. 1998. Dalton's Introduction to Practical Animal Breeding. $4^{\text {th }}$ ed. Blackwell Science, Oxford, UK.

Winder J, J Brinks, R Bourdon, B Golden. 1990. Genetic analysis of absolute growth measurements relative growth rate and restricted selection indices in red Angus cattle. J Anim Sci 68, 330-336.

Yáñez L. 2005. Índice de Selección: Sugerencias para su utilización. En: González-Stagnaro C, Soto-Belloso E (eds). Manual de Ganadería Doble Propósito. Cap. II (8). Ediciones Astro Data S.A. Maracaibo, Venezuela, Pp125-134. 